\title{
Effect of water physical therapy on quality of life in breast cancer survivors
}

\author{
DOI: https://doi.org/10.5114/pq.2018.79741
}

\author{
Tetiana Odynets', Yuriy Briskin', Alina Perederiy ${ }^{1}$, Maryan Pityn', Iryna Svistelnyk² \\ ${ }^{1}$ Department of Theory of Sport and Physical Culture, Lviv State University of Physical Culture, Lviv, Ukraine \\ ${ }^{2}$ Department of Computer Science and Kinesiology, Lviv State University of Physical Culture, Lviv, Ukraine
}

Abstract

Introduction. The purpose of the study was to evaluate the effectiveness of an individualized 12-week water physical therapy program on quality of life parameters in breast cancer survivors.

Methods. Overall, 68 women met the eligibility criteria and were included in the study. They were randomly allocated for the water physical therapy program (experimental group, $n=34$ ) and Pilates physical therapy program (active control group, $n=34$ ). Both groups attended relevant programs for 12 weeks and received 36 rehabilitation sessions. Quality of life parameters were assessed with the questionnaire of the European Organization for Research and Treatment of Cancer (EORTC) with a specific module for breast cancer patients (BR-23). Quality of life data were recorded at baseline and after a 12-week intervention.

Results. In the results analysis, statistically significant differences between the studied groups were identified. The experimental group participants presented significantly fewer negative symptoms associated with the disease and surgical intervention as a whole. Particularly, fatigue was lower by 10.27 points in the experimental group compared with the active control group $(p<0.05)$, insomnia was lower by 13.23 points $(p<0.05)$, appetite loss was smaller by 14.30 points $(p<0.01)$.

Conclusions. The results confirm that a 12-week water physical therapy program effectively improves quality of life parameters, which was reflected in improving general physical condition, shoulder range of motion, emotional and physical functioning, optimism about the future, and a decrease in fatigue, dyspnoea, and insomnia in breast cancer survivors.

Key words: water therapy, breast cancer, quality of life

\section{Introduction}

The problem of studying the quality of life, physical, social, and psychological adaptation of women after surgical treatment for breast cancer becomes more acute as there are more chances of successful recovery in connection with the improvement of long-term results of breast cancer treatment $[1,2]$.

As a result of recent research, it was found that breast cancer survivors had a deterioration in the psycho-emotional status and a decrease in most criteria of quality of life, which were directly related to the severity of post-mastectomy oedema, pain, and neurological disorders [3-6].

Women with breast cancer have an increased risk of developing depression, fear, sexual dysfunction, sleep disturbances, cognitive problems, and fatigue. Life quality parameters have become significant criteria of women's cancer rehabilitation efficiency [7-10].

Physical functioning of women after radical mastectomy deteriorates because of general fatigue, pain, decreased skin sensitivity, limitation of limb movement, and lymphatic oedema [11-13].

In order to increase adaptation to the psycho-traumatic situation and to improve the results of special treatment in breast cancer patients, it is proposed to use non-pharmacological and psychotherapeutic correction during cancer therapy and after its completion [14, 15].

Some studies have shown the most important factors for breast cancer survivors to perform water physical therapy, such as a private dressing room, social interaction, and easily modified weightless exercises [10].

The interest in the possible role of physical exercise in improving the quality of life, reducing the risk of relapse, and increasing the survival rate of cancer patients is increasing today. Physical activity can raise a favourable outlook for cancer biomarkers and energy balance, and contribute to a reduction in the risk of cardiovascular disease, which is one of the major causes of death for many people who survived cancer [7].

A lot of studies have been conducted to improve physical functioning and psychological well-being for breast cancer survivors, including water-based exercises [8-10], yoga exercises [11-13], Pilates intervention [16-18], and yoga combined with meditation [19] for problems associated with poor quality of life among patients.

Taking into account the research data that water exercises are effective and safe methods for these patients, we decided to evaluate the effectiveness of an individualized 12 -week water physical therapy program on quality of life parameters in breast cancer survivors.

\section{Subjects and methods}

The participants' type of surgery treatment and cancer stage were determined with medical records. The research was designed in accordance with the principles of CONSORT. The subjects were randomly allocated for the water physical therapy program (experimental group, $n=34$ ) and

Correspondence address: Tetiana Odynets, Department of Theory of Sport and Physical Culture, Lviv State University of Physical Culture, 79000, Kostiushko Str., 11, Lviv, Ukraine, e-mail: tatyana01121985@gmail.com 
Pilates physical therapy program (active control group, $n=34$ ). The women were randomized into one of these two groups with the use of sequentially numbered, opaque sealed envelopes. Both groups attended relevant programs for 12 weeks and received 36 rehabilitation sessions.

The women had undergone surgical treatment (radical mastectomy by Madden) for breast cancer and stayed in a hospital inpatient department. Radiotherapy was used in addition to the breast cancer surgery. The average time after surgery was $5.62 \pm 0.12$ months. Patients aged 50-60 years with recent history of radical mastectomy, tumour stage 1-2, time after surgery not exceeding 6 months, poor quality of life, absence of metastases, and no contraindications to physical activity were included in the study. The exclusion criteria were the following: bilateral mastectomy, time after surgery more than 6 months, metastases, congestive heart failure, tumour stage 3 , or any contraindications limiting activity.

The study groups were homogenous in terms of demographic and treatment-related characteristics and quality of life parameters at baseline.

Quality of life parameters were assessed with the questionnaire of the European Organization for Research and Treatment of Cancer (EORTC) with a specific module for breast cancer patients (BR-23) [20]. Quality of life data were recorded at baseline and after the 12-week intervention.

EORTC QLQ-C30 is a general self-report questionnaire for cancer patients, containing 30 questions. Indicators for functional and symptomatic scales and individual questions ranged from 0 to 100 points. Thus, a high functional score level reflected high/healthy levels of functioning, but a high symptom score showed a high level of problems. Scoring of the breast cancer module QLQ-BR23 (23 questions) is intended to evaluate systemic therapy side effects, breast symptoms, arm symptoms, body image, and sexual functioning [20]. Applying both questionnaires allows to receive operational information without significant time consuming.

One of the key features of the developed water physical therapy program was rational combination of exercises in the horizontal and vertical positions of the body in deep and shallow water, which helped engage practically all muscles of the body and successfully overcome post-mastectomy complications. The program consisted of a wide range of breathing exercises (static, dynamic) and physical exercises (active, active-passive, special, combined developing, sportsapplied), allowing to solve current tasks. Noodles, blades and water dumbbells were used to increase the load. The intensity of physical activity depended on the motion state and varied from $45 \%$ to $60 \%$ of heart rate reserve.

The water physical therapy program was built on a rational combination of swimming, combined developing exercises, and exercises of local impact on different muscle groups with various initial positions: standing, half-squatting, sitting, lying on noodles. The principle of alternation of physical activity of different muscle groups was observed in order to reduce the manifestations of fatigue and to increase the recovery rate of body functions during the program implementation. Individualization and differentiation of physical exercises in the aquatic and land environment was also carried out with the consideration of the degree of lymphedema. Patients who had lymphedema degree 1-2 performed special exercises from different initial positions: standing, lying on the back, lying on the side, sitting on a fitball. Women with the $3^{\text {rd }}$ degree of lymphedema performed the majority of exercises from lying initial positions without special equipment.
The Pilates program was conducted on the floor and included warm-up, the main part with a resistance band, and cool-down. The total duration of a session was $60 \mathrm{~min}$. Exercise intensity was individualized for each patient and varied from $45 \%$ to $60 \%$ of heart rate reserve. Flexibility and resistance exercises were directed at the muscles of the scapular waist, lower limbs, back, and abdomen.

Data (mean and standard error of the mean) were analysed with the use of the Statistica for Windows (version 8.00) software. Before the statistical analysis, the Shapiro-Wilk test was applied to test for the normal distribution of data. Dependent sample $t$-test was used to analyse life quality changes in one group from baseline to post-intervention. Independent sample $t$-test served to compare life quality between the women of the experimental group and active control group.

\section{Ethical approval}

The research related to human use has been complied with all the relevant national regulations and institutional policies, has followed the tenets of the Declaration of Helsinki, and has been approved by the authors' institutional review board.

\section{Informed consent}

Informed consent has been obtained from all individuals included in this study.

\section{Results}

Demographic and treatment-related characteristics of the studied women are shown in Table 1. These data demonstrated that there were no significant differences between the two intervention groups at baseline. A CONSORT flow diagram is presented in Figure 1.

The results of changes by the functional and symptom scales of the EORTC QLQ-C30 questionnaire in the studied groups are presented in Table 2.

The analysis of self-reported parameters of life quality after therapy indicated that the experimental group partici-

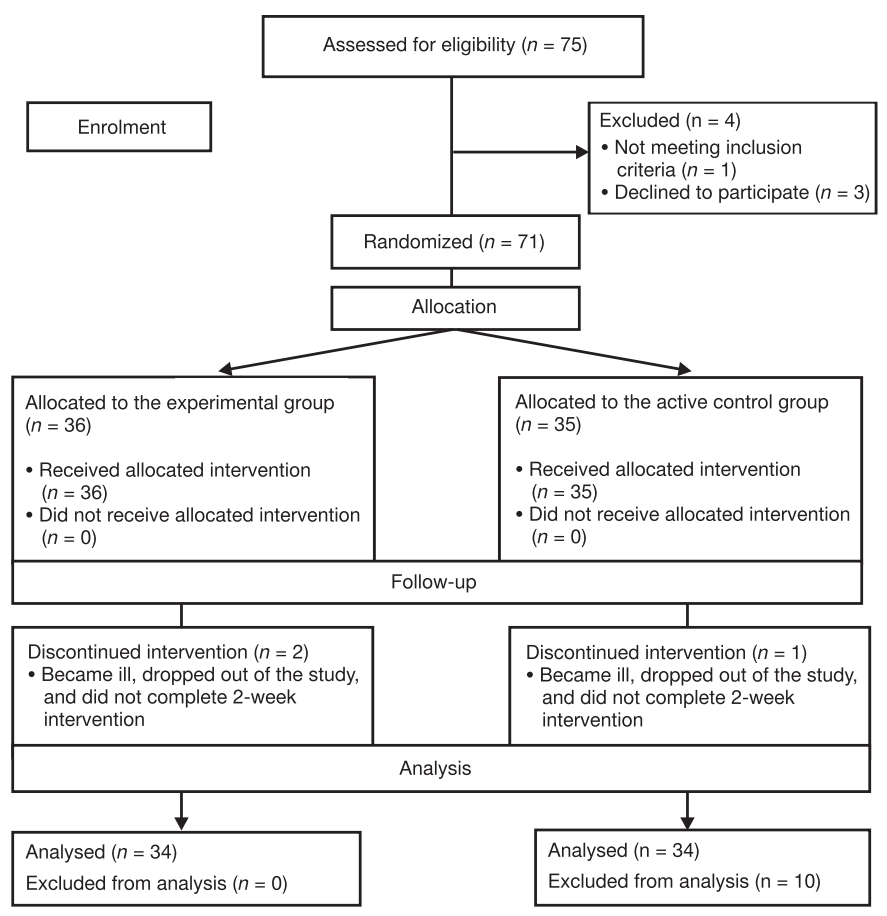

Figure 1. CONSORT flow diagram 
Table 1. Demographic and treatment-related characteristics of the study participants

\begin{tabular}{|c|c|c|c|}
\hline Characteristics & $\begin{array}{l}\text { Experimental group } \\
\qquad(n=34)\end{array}$ & $\begin{array}{l}\text { Active control group } \\
\qquad(n=34)\end{array}$ & $p$ \\
\hline Age $(M \pm m)$ & $57.44 \pm 2.16$ & $57.99 \pm 2.24$ & $>0.05$ \\
\hline \multicolumn{4}{|l|}{ Race } \\
\hline White, $n(\%)$ & $33(97 \%)$ & 32 (94\%) & $>0.05$ \\
\hline Black, $n(\%)$ & $1(3 \%)$ & $2(6 \%)$ & $>0.05$ \\
\hline Married/committed relationship, $n(\%)$ & $28(82 \%)$ & $27(79 \%)$ & $>0.05$ \\
\hline High school graduate, $n(\%)$ & $13(38 \%)$ & $12(35 \%)$ & $>0.05$ \\
\hline College graduate, $n(\%)$ & $19(56 \%)$ & $20(59 \%)$ & $>0.05$ \\
\hline Post-graduate, $n$ (\%) & $2(6 \%)$ & $2(6 \%)$ & $>0.05$ \\
\hline Body mass index, $\mathrm{kg} / \mathrm{m}^{2}(M \pm m)$ & $25.92 \pm 0.42$ & $26.01 \pm 0.81$ & $>0.05$ \\
\hline \multicolumn{4}{|l|}{ Treatment } \\
\hline Surgery type (mastectomy by Madden), $n(\%)$ & $34(100 \%)$ & $34(100 \%)$ & $>0.05$ \\
\hline Time after surgery, months & $5.23 \pm 0.32$ & $5.11 \pm 0.42$ & $>0.05$ \\
\hline \multicolumn{4}{|l|}{ Cancer stage } \\
\hline Stage $1, n(\%)$ & $12(35 \%)$ & $13(38 \%)$ & $>0.05$ \\
\hline Stage 2, $n(\%)$ & 22 (65\%) & $21(62 \%)$ & $>0.05$ \\
\hline
\end{tabular}

$M$ - arithmetic mean, $m$ - error of mean

Table 2. Changes of life quality indicators (arithmetic mean \pm error of mean) in breast cancer survivors by the EORTC QLQ-C30 questionnaire (scores)

\begin{tabular}{|c|c|c|c|c|c|c|}
\hline \multirow[t]{2}{*}{ Indicator } & \multicolumn{2}{|c|}{$\begin{array}{l}\text { Experimental group } \\
\qquad(n=34)\end{array}$} & \multirow[t]{2}{*}{$p$} & \multicolumn{2}{|c|}{$\begin{array}{l}\text { Active control group } \\
\qquad(n=34)\end{array}$} & \multirow[t]{2}{*}{$p$} \\
\hline & Baseline & Post-intervention & & Baseline & Post-intervention & \\
\hline \multicolumn{7}{|l|}{ Functional scale } \\
\hline Physical functioning & $65.67 \pm 1.81$ & $75.80 \pm 2.25$ & $<0.001$ & $69.90 \pm 1.77$ & $71.23 \pm 2.40$ & $>0.05$ \\
\hline Role functioning & $61.47 \pm 2.95$ & $68.20 \pm 2.58$ & $>0.05$ & $59.80 \pm 2.77$ & $62.57 \pm 2.79$ & $>0.05$ \\
\hline Emotional functioning & $58.70 \pm 2.40$ & $76.43 \pm 2.76^{\star \star \star}$ & $<0.001$ & $59.30 \pm 2.69$ & $60.57 \pm 2.61$ & $>0.05$ \\
\hline Social well-being & $60.90 \pm 3.38$ & $68.70 \pm 3.28$ & $<0.05$ & $62.53 \pm 2.81$ & $63.03 \pm 2.26$ & $>0.05$ \\
\hline Cognitive functioning & $61.37 \pm 2.50$ & $75.93 \pm 2.47^{*}$ & $<0.001$ & $61.27 \pm 1.66$ & $67.53 \pm 2.48$ & $<0.05$ \\
\hline Global health status & $64.37 \pm 2.60$ & $77.93 \pm 2.42^{*}$ & $<0.001$ & $64.27 \pm 1.66$ & $65.53 \pm 2.22$ & $>0.05$ \\
\hline \multicolumn{7}{|l|}{ Symptom scale } \\
\hline Fatigue & $44.00 \pm 2.88$ & $30.80 \pm 3.69^{*}$ & $<0.01$ & $44.73 \pm 2.62$ & $41.07 \pm 2.51$ & $>0.05$ \\
\hline Nausea and vomiting & $13.75 \pm 2.68$ & $13.55 \pm 2.88$ & $>0.05$ & $13.15 \pm 2.88$ & $13.15 \pm 2.88$ & $>0.05$ \\
\hline Pain & $45.83 \pm 2.99$ & $27.93 \pm 3.16$ & $<0.001$ & $40.83 \pm 3.93$ & $28.80 \pm 4.36$ & $<0.05$ \\
\hline Dyspnoea & $51.70 \pm 3.48$ & $37.40 \pm 3.85$ & $<0.01$ & $46.20 \pm 4.13$ & $45.10 \pm 3.40$ & $>0.05$ \\
\hline Insomnia & $40.70 \pm 3.48$ & $23.10 \pm 4.30^{*}$ & $<0.01$ & $37.43 \pm 4.19$ & $36.33 \pm 4.07$ & $>0.05$ \\
\hline Appetite loss & $27.50 \pm 3.62$ & $16.50 \pm 3.50^{* *}$ & $<0.05$ & $30.80 \pm 4.53$ & $30.80 \pm 3.91$ & $>0.05$ \\
\hline Constipation & $6.88 \pm 1.68$ & $6.55 \pm 1.27$ & $>0.05$ & $6.15 \pm 2.88$ & $6.25 \pm 1.88$ & $>0.05$ \\
\hline Diarrhoea & $5.78 \pm 1.68$ & $5.75 \pm 1.27$ & $>0.05$ & $6.25 \pm 1.77$ & $6.44 \pm 1.66$ & $>0.05$ \\
\hline
\end{tabular}

${ }^{*} p<0.05,{ }^{* *} p<0.01,{ }^{* * *} p<0.001$ compared with the data between the experimental and active control groups 
pants had significantly better $(p<0.05-0.01)$ indicators by most functional and symptom scales elements in comparison with those in the active control group. At the end of the course, they were more closely aligned with the maximum number of points.

The mean values of indicators for physical functioning were still lagging behind the norm in the study groups, but in the experimental group they significantly improved by 10.13 points $(p<0.001)$ as compared with only 1.33 points improvement $(p>0.05)$ in the active control group.

The patients in the experimental group complained mainly about certain difficulties in the process of performing their work, which requires considerable effort, and also during a long walk. However, no problems with the implementation of a small walk, self-service, or the need to spend a long time in bed were found, while in the patients from the active control group these complaints were preserved at the end of the rehabilitation course.

The women after breast cancer surgery still experienced some limitations during day-to-day operations or leisure; in particular, $53 \%$ of the subjects in the experimental group and $63 \%$ in the active control group suffered from mild difficulties, and $17 \%$ and $27 \%$, respectively, experienced significant difficulties; the rest did not have any limitations.

The most significant improvement was observed in the subscale of emotional functioning. These indicators significantly increased by 17.73 points $(p<0.001)$ in the experimental group compared with 1.27 points $(p<0.05)$ in the active control group.

Feelings of tension, anxiety, irritation, depression were significantly reduced in women after performing the water physical therapy program.

Estimating the results of the questionnaire in the subscale of social functioning, we found a significant improvement by 7.80 points $(p<0.05)$ in the experimental group women, while in the active control group only a positive dynamics of this indicator was observed. Women still had some manifestations of the sense of inferiority of their physical condition during their appearance in public, as well as encumbrance of family members with their condition after surgery.

The analysis of self-reported indicators by the symptom scale of EORTC QLQ-C30 showed that in patients of the experimental group fatigue decreased by 13.20 points $(p<0.01)$, pain decreased by 17.90 points $(p<0.001)$, dyspnoea de- creased by 14.30 points $(p<0.01)$, insomnia decreased by 17.60 points $(p<0.01)$, and appetite loss decreased by 11.00 points $(p<0.05)$ after the 12 -week intervention.

A detailed analysis of dyspnoea proved that weak dyspnoea was reported by $60 \%$ of the experimental group patients, moderate dyspnoea by $27 \%$, no dyspnoea by $13 \%$. At the same time, in the active control group, poor dyspnoea was observed in $57 \%$, and moderate in $40 \%$ of the respondents.

As for the summary results of the symptom scores by the EORTC QLQ-C30 questionnaire in all groups after the course of physical rehabilitation, it was identified that the participants of the experimental group presented significantly fewer negative symptoms associated with the disease and surgical intervention as a whole. Particularly, fatigue decreased by 10.27 points in the experimental group compared with the active control group $(p<0.05)$, insomnia by 13.23 points $(p<$ $0.05)$, pain by 0.87 points $(p>0.05)$, and appetite loss by 14.30 points $(p<0.01)$.

On the basis of the results of the survey with a specific QLQ-BR23 module (Table 3), we established certain significant differences in the study groups after performing the rehabilitation programs. Particularly, fear, feelings about the body image, and the sense of femininity loss significantly decreased in both groups, which was reflected in an improvement of body image by 9.80 points $(p<0.01)$ in the experimental group and by 7.23 points $(p<0.01)$ in the active control group.

Analysing the answers to the question about future perspectives, we found that the concerns about health in the future significantly decreased in the experimental group women, while in those in the active control group the value of this indicator remained almost unchanged.

As can be concluded from Table 3, the average results with regard to future perspectives were statistically significantly better in the experimental group subjects compared with the active control group by 14.47 points $(p<0.05)$; breast symptoms improved by 9.67 points $(p<0.05)$, respectively.

\section{Discussion}

The research results indicate that the implementation of the water physical therapy program had a substantial influence on life quality in women after breast cancer surgery. The 12-week monitoring demonstrated that applying water

Table 3. Changes of life quality indicators (arithmetic mean \pm error of mean) in breast cancer survivors by the QLQ-BR23 questionnaire (scores)

\begin{tabular}{|c|c|c|c|c|c|c|}
\hline \multirow{2}{*}{ Indicator } & \multicolumn{2}{|c|}{$\begin{array}{l}\text { Experimental group } \\
\qquad(n=34)\end{array}$} & \multirow{2}{*}{$p$} & \multicolumn{2}{|c|}{$\begin{array}{l}\text { Active control group } \\
\qquad(n=34)\end{array}$} & \multirow{2}{*}{$p$} \\
\hline & Baseline & Post-intervention & & Baseline & Post-intervention & \\
\hline Body image & $55.30 \pm 2.65$ & $65.10 \pm 2.56$ & $<0.01$ & $56.40 \pm 2.67$ & $63.63 \pm 1.88$ & $<0.01$ \\
\hline Sexual functioning & $7.13 \pm 2.50$ & $8.23 \pm 2.63$ & $>0.05$ & $10.97 \pm 2.82$ & $12.10 \pm 3.00$ & $>0.05$ \\
\hline Sexual enjoyment & $4.40 \pm 2.11$ & $5.50 \pm 2.82$ & $>0.05$ & $9.90 \pm 3.27$ & $12.10 \pm 3.40$ & $>0.05$ \\
\hline Future perspectives & $53.77 \pm 4.45$ & $67.00 \pm 4.25^{*}$ & $<0.01$ & $51.60 \pm 3.10$ & $52.53 \pm 5.06$ & $>0.05$ \\
\hline Breast symptoms & $32.70 \pm 3.25$ & $21.93 \pm 3.57^{*}$ & $<0.001$ & $39.27 \pm 2.92$ & $31.60 \pm 2.42$ & $<0.05$ \\
\hline Arm symptoms & $43.27 \pm 2.83$ & $31.90 \pm 2.41$ & $<0.001$ & $43.63 \pm 1.81$ & $36.30 \pm 3.08$ & $<0.05$ \\
\hline Upset by hair loss & $13.75 \pm 2.54$ & $13.55 \pm 2.88$ & $>0.05$ & $13.15 \pm 2.88$ & $12.19 \pm 2.18$ & $>0.05$ \\
\hline Systemic therapy side effects & $22.75 \pm 2.68$ & $13.15 \pm 1.88$ & $<0.05$ & $23.15 \pm 1.98$ & $19.15 \pm 2.88$ & $>0.05$ \\
\hline
\end{tabular}


exercises was more effective compared with land exercises for improvement the quality of life in breast cancer survivors at the outpatient treatment stage.

The study supports a considerable body of research that shows a significant role of regular physical exercises in reducing stress, fatigue, depression, as well as improving physical fitness, life quality, and specific quality of life domains in breast cancer survivors [1, 4, 6, 8, 12, 21, 22].

Numerous studies have confirmed that water exercises are valuable tools for reducing pain and lymphedema, but various intervention methods, duration, and intensity of exercises create some difficulties in comparing the results obtained in the context of the current research [4, 8-10].

Some studies analysed aquatic therapy programs and patient satisfaction in women after breast cancer surgery $[3,4,9]$, other investigations [16-18] focused on the analysis of Pilates exercises as useful practices for women experiencing lymphedema, fatigue, and sleep disturbance. Previous studies applied the interventions frequencies of 2-3 sessions per week, durations of 4-8 weeks, with different combinations of intensity for breast cancer patients.

This research results indicate that swimming, special exercises for coordination, increasing the shoulder range of motion, balance, and stretching contribute to the improvement in physical condition and quality of life in breast cancer survivors. Dynamic water physical exercises in different planes of motion, as well as special exercises for increasing muscle strength with the use of noodles and swimming boards were applied in the program. After practicing the water physical program, the women began to feel more powerful, efficient, full of strength, resilient, and vigorous, which affected their well-being and ability to perform daily activities.

The strength of our current research consists in providing a rational combination of breathing exercises, special exercises in the horizontal and vertical positions of the body in deep and shallow water, as well as applying noodles and dumbbells, which allowed to engage practically all muscles of the body and successfully overcome post-mastectomy complications.

\section{Limitations}

The weak side of our study is that the physical, role-related, and emotional functioning, as well as social well-being were evaluated with a questionnaire, and the patients' lifestyle differences could have an impact on the obtained results. Moreover, the sample was not more than 6 months after surgery, and race was mostly white, so it would be hard to extrapolate the results to other races and periods after surgical intervention.

\section{Conclusions}

The results of the study have confirmed that the 12-week water physical therapy program was effective for improving quality of life parameters in breast cancer survivors. The developed program positively influenced the global quality of life in women, which was reflected in the improvement of the general physical condition, shoulder range of motion, emotional and physical functioning, optimism about the future, and a decrease in fatigue, dyspnoea, and insomnia.

Further research should evaluate the effectiveness of an individualized 12-week yoga physical therapy program on quality of life parameters in breast cancer survivors.

\section{Disclosure statement}

No author has any financial interest or received any financial benefit from this research.

\section{Conflict of interest}

The authors state no conflict of interest.

\section{References}

1. Cuesta-Vargas Al, Buchan J, Arroyo-Morales M. A multimodal physiotherapy programme plus deep water running for improving cancer-related fatigue and quality of life in breast cancer survivors. Eur $\mathrm{J}$ Cancer Care. 2014; 23(1):15-21; doi: 10.1111/ecc.12114.

2. Odynets T, Briskin Y, Sydorko O. Psycho-emotional state and quality of life characteristics in women with postmastectomy syndrome with different types of attitude to the disease. Physiother Quart. 2018;26(1):9-12; doi: 10.5114/pq.2018.74706.

3. Johansson K, Hayes S, Speck RM, Schmitz KH. Waterbased exercise for patients with chronic arm lymphedema: a randomized controlled pilot trial. Am J Phys Med Rehabil. 2013;92(4):312-319; doi: 10.1097/PHM.0b013e $318278 \mathrm{b0e8}$.

4. Lindquist H, Enblom A, Dunberger G, Nyberg T, Bergmark K. Water exercise compared to land exercise or standard care in female cancer survivors with secondary lymphedema. Lymphology. 2015;48(2):64-79.

5. Battaglini CL, Mills RC, Phillips BL, Lee JT, Story CE, Nascimento MG, et al. Twenty-five years of research on the effects of exercise training in breast cancer survivors: a systematic review of the literature. World J Clin Oncol. 2014;5(2):177-190; doi: 10.5306/wjco.v5.i2.177.

6. Mehnert A, Veers S, Howaldt D, Braumann KM, Koch U, Schulz KH. Effects of a physical exercise rehabilitation group program on anxiety, depression, body image, and health-related quality of life among breast cancer patients. Onkologie. 2011;34(5):248-253; doi:10.1159/000327813.

7. Valenti M, Porzio G, Aielli F, Verna L, Cannita K, Manno R, et al. Physical exercise and quality of life in breast cancer survivors. Int J Med Sci. 2008;5(1):24-28; doi: 10.7150/ ijms.5.24.

8. Cantarero-Villanueva I, Fernández-Lao C, Cuesta-Vargas AI, Del Moral-Avila R, Fernández-de-Las-Peñas C, Arroyo-Morales M. The effectiveness of a deep water aquatic exercise program in cancer-related fatigue in breast cancer survivors: a randomized controlled trial. Arch Phys Med Rehabil. 2013;94(2):221-230; doi: 10.1016/j.apmr.2012.09.008.

9. Cantarero-Villanueva I, Fernández-Lao C, Fernándezde-Las-Peñas C, López-Barajas IB, Del-Moral-Ávila R, de la-Llave-Rincón Al, et al. Effectiveness of water physical therapy on pain, pressure pain sensitivity, and myofascial trigger points in breast cancer survivors: a randomized, controlled clinical trial. Pain Med. 2012;13(11): 1509-1519; doi: 10.1111/j.1526-4637.2012.01481.x.

10. Enblom A, Lindquist $H$, Bergmark K. Participation in water-exercising long-term after breast cancer surgery: experiences of significant factors for continuing exercising as a part of cancer rehabilitation. Eur $\mathrm{J}$ Cancer Care. 2018;27(1); doi: 10.1111/ecc.12736.

11. Levine AS, Balk JL. Pilot study of yoga for breast cancer survivors with poor quality of life. Complement Ther Clin Pract. 2012;18(4):241-245; doi: 10.1016/j.ctcp.2012. 06.007. 
12. Levine AS, Balk JL. Yoga and quality-of-life improvement in patients with breast cancer: a literature review. Int J Yoga Therap. 2012;22:95-99.

13. Ulger $O$, Yağli NV. Effects of yoga on the quality of life in cancer. Complement Ther Clin Pract. 2010;16(2):60-63; doi: 10.1016/j.ctcp.2009.10.007.

14. Li XM, Zhou KN, Yan H, Wang DL, Zhang YP. Effects of music therapy on anxiety of patients with breast cancer after radical mastectomy: a randomized clinical trial. J Adv Nurs. 2012;68(5):1145-1155; doi: 10.1111/j.13652648.2011.05824.x.

15. Yilmaz SG, Arslan S. Effects of progressive relaxation exercises on anxiety and comfort of Turkish breast cancer patients receiving chemotherapy. Asian Pac J Cancer Prev. 2015;16(1):217-220; doi: 10.7314/APJCP.2015. 16.1.217.

16. Stan DL, Rausch SM, Sundt K, Cheville AL, Youdas JW, Krause DA, et al. Pilates for breast cancer survivors. Clin J Oncol Nurs. 2012;16(2):131-141; doi: 10.1188/12.CJON. 131-141.

17. Şener HO, Malkoç M, Ergin G, Karadibak D, Yavuzşen T. Effects of clinical Pilates exercises on patients developing lymphedema after breast cancer treatment: a randomized clinical trial. J Breast Health. 2017;13(1):1622; doi: 10.5152/tjbh.2016.3136.

18. Keays KS, Harris SR, Lucyshyn JM, Maclntyre DL. Effects of Pilates exercises on shoulder range of motion, pain, mood, and upper-extremity function in women living with breast cancer: a pilot study. Phys Ther. 2008;88(4): 494-510; doi: 10.2522/ptj.20070099.

19. Cramer H, Rabsilber S, Lauche R, Kümmel S, Dobos G. Yoga and meditation for menopausal symptoms in breast cancer survivors - a randomized controlled trial. Cancer. 2015;121(13):2175-2184; doi: 10.1002/cncr.29330.

20. EORTC QLQ-C30 (Quality of Life Questionnaire). Available from: http://groups.eortc.be/qol/eortc-qlq-c30.

21. Zeng Y, Huang M, Cheng AS, Zhou Y, So WK. Metaanalysis of the effects of exercise intervention on quality of life in breast cancer survivors. Breast Cancer. 2014; 21(3):262-274; doi: 10.1007/s12282-014-0521-7.

22. Phillips SM, Awick EA, Conroy DE, Pellegrini CA, Mailey EL, McAuley E. Objectively measured physical activity and sedentary behavior and quality of life indicators in survivors of breast cancer. Cancer. 2015;121(22): 4044-4052; doi: 10.1002/cncr.29620. 